\title{
Epitaxial Hybrid Ferromagnet-Semiconductor Structures: Growth, Structural and Magnetic Properties
}

\author{
L. Däweritz \\ Paul Drude Institute for Solid State Electronics, Hausvogteiplatz, 5-7, D-10117 Berlin, Germany
}

Received on 9 March, 2003

\begin{abstract}
Ferromagnet-semiconductor heterostructures are promising materials for the integration of magnetic or spin related functions into semiconductor materials and devices. Fe-on-GaAs and MnAs-on-GaAs are canditates for potential room temperature applications. We review our recent results on growth, structural and magnetic properties of these materials, and their use as spin injectors. The most critical issue of $\mathrm{Fe} / \mathrm{GaAs}$ heterostructures is the delicate interface formation during epitaxy and its possible modification during subsequent processing. The properties of the MnAs/GaAs heterostructures are intimately related to the phase transition between ferromagnetic and paramagnetic phases.
\end{abstract}

\section{Introduction}

The introduction of magnetic or spin related functions into semiconductor materials and devices became a subject of intense research over the past few years [1-3]. In particular, the desire to use not only the charge but also the spin of electrons for the development of novel devices motivated work about monolithic integration of ferromagnetic and semiconductor materials using ultra-high vacuum growth techniques. A key of success is the epitaxial growth of heterostructures with well-ordered heterointerfaces which allow spin injection from the ferromagnetic layer into the semiconductor. Heterostructures of high structural quality can be realized in particular for the case of low lattice mismatch between similar materials. However, layer structures like $\mathrm{Ga}_{1-x} \mathrm{Mn}_{x} \mathrm{As}$ on GaAs exhibit a Curie temperature being far below room temperature [4]. Two promising material systems for spin injection at room temperature are $\mathrm{Fe}$ on-GaAs and MnAs-on-GaAs. Epitaxial growth of such dissimilar materials encounters specific problems, like the thermodynamic stability of the interface, large anisiotropic misfit, and phase transitions. In this article, we review our recent results on low-temperature growth of Fe-on-GaAs and MnAs-on-GaAs heterostructures by molecular beam epitaxy (MBE), their structural and magnetic properties, as well as the successful use of the ferromagnetic layers as spin injectors.

\section{Results and Discussion}

\section{A. Fe-on-GaAs}

$\mathrm{Fe}-\mathrm{on}-\mathrm{GaAs}$ is the most widely investigated epitaxial ferromagnetic metal-semiconductor hybrid system [5-7]. The stable bcc Fe phase, with its bulk lattice constant being nearly half that of GaAs $\left(2 a_{F e} / a_{G a A s}=1.012\right)$, grows in
(001) orientation on $\mathrm{GaAs}(001)$ with $\mathrm{Fe}[100]$ || GaAs[100]. In the majority of investigations $\mathrm{Fe}$ has been deposited at elevated temperatures $\left(150-180^{\circ} \mathrm{C}\right)$. Under such conditions interfacial reactions occur with detrimental consequences for the ferromagnetic properties of the first few nanometers. However, no indications for the formation of $\mathrm{Fe}_{x} \mathrm{Ga}_{y} \mathrm{As}_{z}$ alloys were found by x-ray diffraction in 20-nm-thick films at a growth temperature of $50^{\circ} \mathrm{C}$ in contrast to the case of $175^{\circ} \mathrm{C}$ growth temperature [8]. Such films grow by quasitwodimensional nucleation and coalescence of nanometerscale islands.

Recently, the ferromagnetic properties of ultrathin layers grown at $50^{\circ} \mathrm{C}$ were studied [9]. For layer thicknesses $<10$ monolayers (ML) the in-plane magnetization of such films exhibits a strong uniaxial anisotropy with the easy and hard axis of magnetization parallel to GaAs[110] and GaAs [110], respectively. With decreasing film thickness a strong reduction of the Curie temperature is observed, which could originate from the decreasing number of neighbouring atoms. Ferromagnetic behavior at room temperatur is observed in films as thin as $3.5 \mathrm{ML}$. The saturation magnetization of films with $d_{F e} \geq 4 \mathrm{ML}$ for $T \rightarrow 0 \mathrm{~K}$ is found to be close to the value of bulk bcc $\mathrm{Fe}(100)$ which amounts to $1740 \mathrm{emu} / \mathrm{cm}^{3}$.

20-nm-thick Fe films were successfully used as spin injection layer [10]. Room temperature injection of spinpolarized electrons into an inverted $n-i-p(\mathrm{Ga}, \mathrm{In}) \mathrm{As}$ light emitting diode (LED) was demonstrated by measuring the degree of the circular polarization of the emitted electroluminescence light. The active region of the MBE-grown LED is comprised of two 4-nm-Ga ${ }_{0.8} \mathrm{In}_{0.2}$ As quantum wells separated by a $10-\mathrm{nm}-\mathrm{GaAs}$ spacer layer. On top of this intrinsic region, a 70-nm- $n$-doped GaAs layer was grown. The Fe injection layer was deposited on this $n$-GaAs in an As-free MBE chamber attached to the III-V MBE chamber. The effective polarization degree of the optical signal from the LED as function of the external field follows the 
independently measured out-of-plane magnetization of the Fe layer. This demonstrates successful spin injection with an efficiency of $\approx 5 \%[10,11]$. The observed spin injection from a ferromagnetic metal into a semiconductor can be explaind by tunneling through a Schottky barrier at the interface [12].

\section{B. MnAs-on-GaAs}

Bulk MnAs is ferromagnetic with a hexagonal NiAs structure below $\approx 40^{\circ} \mathrm{C}$, where it experiences a first-order phase transition to a paramagnetic $\beta$-MnAs phase with an orthorhombic structure. A further transition occurs at $\approx 125^{\circ} \mathrm{C}$ to paramagnetic $\gamma$-MnAs, which has again a NiAs structure [13].

Ferromagnetic MnAs can be grown epitaxially on $\mathrm{GaAs}(001)$, although the lattice mismatch is very large and anisotropic, i.e., 7.5\% along the GaAs[110] direction and about $30 \%$ along the GaAs[110] direction $[14,15]$ (see Fig. 1). Two fundamental issues for understanding this unexpected result of real heteroepitaxy have been identified: (i) an interesting anisotropic lattice mismatch accommodation mechanism [16] and (ii) the strain-mediated coexistence of $\alpha$ - and $\beta$-MnAs below the bulk phase transformation temperature [17].

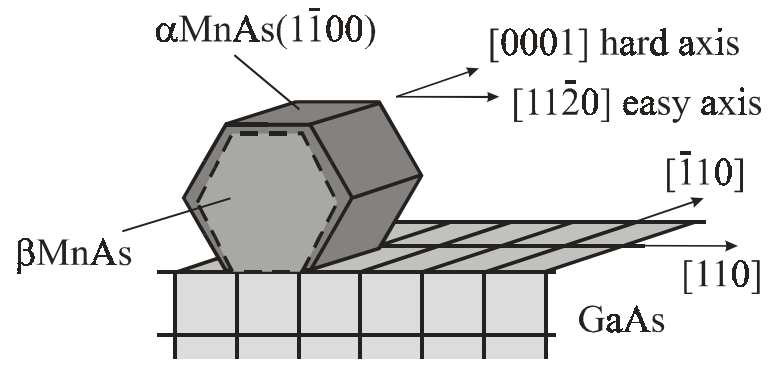

Figure 1. Scheme of the epitaxy of MnAs on GaAs(001) and orientation of the hard and easy axis of magnetization.

By controlling the stoichiometry of the GaAs template and the MnAs growth conditions, several epitaxial orientations can be realized $[14,15]$. Our films were grown at conditions (substrate temperature $250^{\circ} \mathrm{C}, \mathrm{As}_{4}$ : $\mathrm{Mn}$ beam equivalent pressure ratio of 90 , growth rate $20 \mathrm{~nm} \mathrm{~h}^{-1}$ ) leading to the unique A-orientation (Fig. 1). The $\operatorname{MnAs}(1 \overline{1} 00)$ plane is parallel to $\mathrm{GaAs}(001)$ and the MnAs[0001] direction, which is also the hard axis of magnetization, is parallel to GaAs[110]. The easy axis of magnetization lies also inplane along the $\mathrm{MnAs}[11 \overline{2} 0]$ direction which is parallel to GaAs[110].

As observed in situ by reflection high-energy electron diffraction (RHEED) and confirmed by scanning tunneling microscopy, in early growth stages three-dimensional islands exist before after deposition of about $5 \mathrm{ML}$ due to coalescence a smooth film develops [18]. Further growth on such a surface proceeds layer-by-layer as evidenced by RHEED intensity oscillations [19]. High-resolution transmission electron microscopy studies show that the $\mathrm{MnAs} / \mathrm{GaAs}$ interface is smooth and chemically sharp [16].
In thicker films, in addition to the $\alpha$-phase of MnAs the $\beta$-phase is detected by $x$-ray diffraction. In Fig. 1 the unit cell of the orthorhombic $\beta$-phase is shown using a dashed line. At the $\beta \rightarrow \alpha$ phase transition during cooling from growth to room temperature, the plane corresponding to (0001) of the $\alpha$-phase expands discontinously by $\approx 1.2 \%$, whereas the change along [0001] is comparatively small [20]. The epitaxial constraints of the film lead to an equilibrium coexistence between $\alpha$ - and $\beta$-MnAs over a wide temperature interval [17]. The phase composition of the films is obtained from $\omega-2 \theta \mathrm{x}$-ray diffraction scans which show two separate [1100] and (020) peaks for the $\alpha$ - and $\beta$ phase, respectively. The ratio of their integrated intensities gives the fraction of $\alpha$-MnAs. Fig. 2(a) presents data for heating and cooling of a 180-nm-thick film. The fraction of $\alpha$-MnAs almost linearly increases upon cooling in a temperature interval of more than $20 \mathrm{~K}$. The phase coexistence is accompanied by a temperature hysteresis.

As a result of the epitaxial strain induced at the $\beta \rightarrow \alpha$ phase transition, a striped phase separation occurs $[17,21]$. A self-organized pattern with a periodic array of ridges and grooves is observed at room temperature (Fig. 3(a)). The groove direction is along MnAs[0001]. The ridges consist of $\alpha$-MnAs and the grooves of $\beta$-MnAs.

To give direct evidence for the assignment of the ridges to the ferromagnetic $\alpha$-phase, we employed magnetic force microscopy (MFM) [22]. By varying the working distance of the cantilever, images with topographical and magnetic contrast of the same area are obtained. Magnetic contrast as meander-like structure is found only in the area of the ridges. In neighbouring domains, the magnetic moments are oriented opposite to each other, i.e., along [112 0 ] and [1120], respectively. Since [0001] is the hard axis of magnetization and the out-of-plane [1]100] direction is a medium axis of magnetization, the rotation of the magnetic moment occurs via an out-of-plane rotation, giving rise to the observed magnetic contrast [23]. This meander-like contrast is due to domain walls of the Bloch-type.

The dynamics of the phase transition has been studied by employing temperature-dependent scanning probe microscopy to image the topography [24]. The development of a rippled surface morphology with a periodic arrangement of ridges and grooves is observed in the temperaure range from about $40^{\circ} \mathrm{C}$ to $12^{\circ} \mathrm{C}$. While the ridges, which appear just below the Curie temperature of MnAs, are initially in an irregular arrangement, an ordereing process takes place at lower temperature. The width of the ridges increases upon cooling, until finally the grooves are completely closed.

The magnetic phase transition has been investigated by measuring the temperature dependence of the magnetization by SQUID magnetometry, without magnetic field (Fig. 2(b)) and with a magnetic field of 1000 Oe applied along the easy axis of magnetization (Fig. 2(c)). The spontaneous magnetization shows a pronounced temperature hysteresis. We first consider the heating branch of Fig. 2(b). Upon elevating the temperature, the spontaneous magnetization of the $\alpha$-MnAs decreases gradually until at $\approx 280 \mathrm{~K}$ the change becomes stronger due to the beginning coexistence with the $\beta$ phase (cf. the X-ray data in Fig. $2(\mathrm{a})$ ). At $\approx 300 \mathrm{~K}$ there 
is an abrupt disappearance of magnetization due to splitting into domains. It is not discontinuous as expected for an ideal first-order phase transition from the ferromagnetic to the paramagnetic state [25]. In agreement with the phase composition (cf. Fig. 2(a)), the spontaneous magnetization becomes zero at $\approx 315 \mathrm{~K}$. Thus, the heating branches of the magnetization curve and of the phase composition curve are strongly correlated and the apparent deviation from a firstorder magnetic transition is due to the strain-mediated phase coexistence of $\alpha$ - and $\beta$-MnAs.
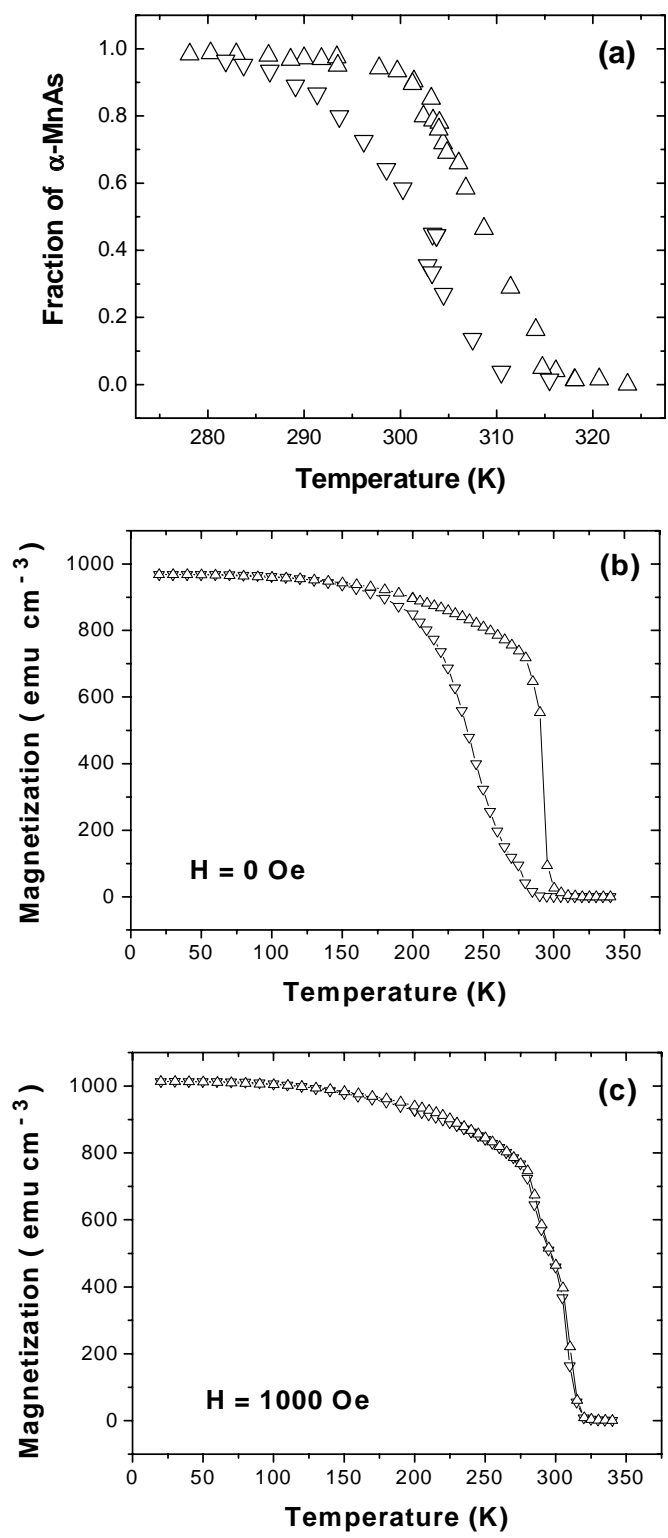

Figure 2.(a) Fractions of $\alpha$-MnAs obtained on heating and cooling of a 180-nm-thick MnAs film. Temperature dependence of the magnetization measured without external magnetic field (b) and with applying a field of 1000 Oe (c). Heating and cooling branches are indicated by triangles up and down, respectively.

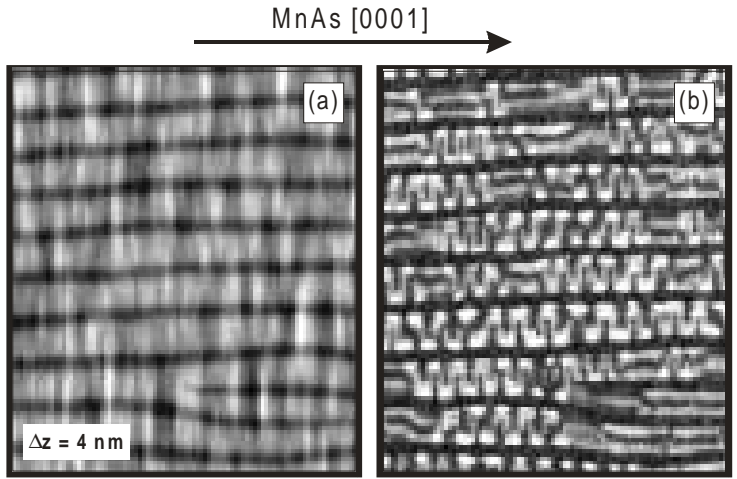

Figure 3. MFM images of a 180-nm-thick MnAs film. Both images represent the same scanning area $(6 \mu \mathrm{m} \times 7 \mu \mathrm{m})$ with topographyinduced contrast (a) and contrast induced by magnetic signal (b).

Upon cooling, the magnetization is not re-established at $280 \mathrm{~K}$ (Fig. 2(b)) although the film consists already completely of $\alpha$-MnAs (Fig. 2(a)). The cooling branch matches the heating branch not before a temperature as low as $\approx 150$ $\mathrm{K}$ is reached.

Insight into the large asymmetry of this ferromagneticparamagnetic phase transition provides a recent study of the MnAs films with x-ray magnetic circular dichroism photoemission electron microscopy (XMCDPEEM) [26]. As schematically shown in Fig. 4(a), the XMCDPEEM image of a film in the $\alpha \rightarrow \beta$ transition stage upon heating from below $273 \mathrm{~K}$ reveals ferromagnetic domains, which are strongly elongated in [0001] direction. In neighbouring domains the magnetic moments are oriented opposite to each other. The paramagnetic stripes perpendicular to the easy axis break the domains up into narrow stripes, which are still well correlated. In a MnAs film cooled from the completely paramagnetic state to room temperature there is yet mainly ferromagnetic coupling between the neighbouring ferromagnetic stripes. But many oppositely magnetized regions occur, too, as sketched in Fig. 4(b).

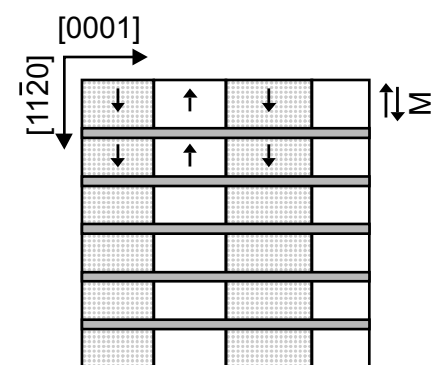

(a)

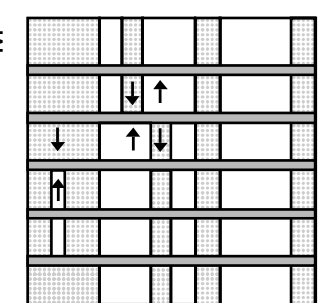

(b)
Figure 4. Schematic representation of the array of magnetic domains in the coexistence range of the $\alpha$ - and $\beta$-phase of a MnAs film upon heating from below $273 \mathrm{~K}$ (a) and upon cooling from the completely paramagnetic state (b).

Applying a field of 1000 Oe along the easy axis of magnetization (Fig. 2(c)), the heating and cooling branches of the magnetization curve coincide, and a Curie temperature of $317 \mathrm{~K}$ is found. The behavior of the magnetization curve 
correlates with that of the heating branch of the phase composition curve, suggesting that the hysteresis of the composition curve can be lifted by applying a magnetic field.

50-nm-thick MnAs layers were used for spin injection at $80 \mathrm{~K}$ [11]. The electrical spin injection from MnAs into GaAs was again studied by means of the circular polarization of electroluminescent light from an an inverted $n-i-p$ $(\mathrm{Ga}, \mathrm{In}) \mathrm{As}$ LED in the same configuration as described for the Fe-on-GaAs system. A spin injection efficiency of about $6 \%$ was obtained.

\section{Conclusion}

Ferromagnetic $\mathrm{Fe}$ and MnAs layers with high-quality interfaces can be grown by MBE on $\operatorname{GaAs}(001)$. Utilizing the optical signal from a $(\mathrm{Ga}, \mathrm{In}) \mathrm{As}$ LED spin injection from both $\mathrm{Fe}$ and MnAs into GaAs with an efficiency of about $5 \%$ was abserved at room temperature and at $80 \mathrm{~K}$, respectively. Most of the properties of the Fe-on-GaAs heterostructures can directly be correlated with the intricate ferromagnetsemiconductor interface formation during growth and modification during subsequent processing. MnAs-on-GaAs heterostructures, on the other hand, have a high thermal stability but show a complex structural and magnetic behavior. In particular, a phase separation process during cooling after growth leads to an interesting self-organized pattern with alternating stripes of ferromagnetic $\alpha$-MnAs and paramagnetic $\beta$-MnAs.

\section{Acknowledgement}

The author wishes to thank K. H. Ploog for continuous support and the colleagues C. Herrmann, T. Hesjedal, B. Jenichen, M. Kästner (now at European Patent Office, H V Rijswijk, The Netherlands), and T. Plake for active contributions to this work. Part of this work was sponsored by the Bundesministerium für Bildung and Forschung.

\section{References}

[1] G. A. Prinz, Science 250, 1092 (1990).

[2] G. A. Prinz, Phys. Today 48, 58 (1995).

[3] M. Tanaka, Semicon. Sci. Technol. 17, 327 (2002).

[4] Y. Ohno, D. K. Young, B. Beschoten, F. Matsukara, H. Ohno, and D. D. Awschalom, Nature (London) 402, 790 (1999).

[5] J. J. Krebs, T. Jonker, and G. A. Prinz, J. Appl. Phys. 61, 2596 (1987).
[6] E. M. Kneedler, B. T. Jonker, P. M. Thibado, R. J. Wagner, B. V. Shanabrook, and L. J. Whitman, Phys. Rev. B 56, 8163 (1997).

[7] Y. B. Xu, E. T. M. Kernohan, D. J. Freeland, A. Ercole, M. Tselepi, and J. A. C. Bland, Phys. Rev. B 58, 890 (1998).

[8] H.-P. Schönherr, R. Nötzel, W. Q. Ma, and K. H. Ploog, J. Appl. Phys. 89, 169 (2001).

[9] K. H. Ploog, J. Herfort, H.-P. Schönherr, M. Moreno, S. Dhar, J. Crystal Growth 251, 292 (2003).

[10] H. J. Zhu, M. Ramsteiner, H. Kostial, M. Wassermeier, H.P. Schönherr, and K. H. Ploog, Phys. Rev. Lett. 87, 016601 (2001).

[11] M. Ramsteiner, H. Y. Hao, A. Kawaharazuka, H. J. Zhu, M. Kästner, R. Hey, L. Däweritz, H. T. Grahn, and K. H. Ploog, Phys. Rev. B 66, 081304 (2002).

[12] E. I. Rashba, Phys. Rev. B 62, R16267 (2000).

[13] H. Okamoto, Bull. Alloy Phase Diagrams 10, 549 (1989).

[14] M. Tanaka, J. P. Harbison, M. C. Park, T. Shin, and G. M. Rothberg, J. Appl. Phys. 76, 6278 (1994).

[15] F. Schippan, A. Trampert, L. Däweritz, and K. H. Ploog, J. Vac. Sci. Technol. B 17, 1716 (1999).

[16] A. Trampert, F. Schippan, L. Däweritz, and K. H. Ploog, Appl. Phys. Lett. 78, 2461 (2001).

[17] V. M. Kaganer, B. Jenichen, F. Schippan, W. Braun, L. Däweritz, and K. H. Ploog, Phys. Rev. B 66, 045305 (2002).

[18] M. Kästner, F. Schippan, P. Schützendübe, L. Däweritz, and K. H. Ploog, J. Vac. Sci. Technol. B 18, 2052 (2000).

[19] F. Schippan, M. Kästner, L. Däweritz, and K. H. Ploog, Appl. Phys. Lett. 76, 834 (2000).

[20] B. T. M. Willis and H. P. Rooksby, Proc. Phys. Soc. (London) B 67, 290 (1954).

[21] M. Kästner, C. Herrmann, L. Däweritz, and K. H. Ploog, J. Appl. Phys. 92, 5711 (2002).

[22] L. Däweritz, M. Kästner, T. Hesjedal, T. Plake, B. Jenichen, and K. H. Ploog, J. Crystal Growth 251, 297 (2003).

[23] F. Schippan, G. Behme, L. Däweritz, K. H. Ploog, B. Dennis, K.-U. Neumann, and K. R. A. Ziebeck, J. Appl. Phys. 88, 2766 (2000).

[24] T. Plake, M. Ramsteiner, V. M. Kaganer, B. Jenichen, M. Kästner, L. Däweritz, and K. H. Ploog, Appl. Phys. Lett. 80, 2523 (2002).

[25] C. P. Bean and D. S. Rodbell, Phys. Rev. 126, 104 (1962).

[26] E. Bauer, S. Cherifi, L. Daeweritz, M. Kaestner, S. Heun, and A. Locatelli, J. Vac. Sci. Technol. B 20, 2539 (2002). 